\title{
The algorithm of adaptive control for active suspension systems using pole assign and cascade design method
}

\author{
Chi Nguyen Van \\ Thai Nguyen University of Technology, Vietnam
}

\begin{tabular}{l} 
Article Info \\
\hline Article history: \\
Received Mar 11, 2020 \\
Revised May 31, 2020 \\
Accepted Sep 10, 2020 \\
\hline
\end{tabular}

\section{Keywords:}

Adaptive control

Cascade control

Pole assignment method

Road excitation

Road profile

Suspension system

\begin{abstract}
This paper presents the active suspension system (ASS) control method using the adaptive cascade control scheme. The control scheme is implemented by two control loops, the inner control loop and outer control loop are designed respectively. The inner control loop uses the pole assignment method in order to move the poles of the original system to desired poles respect to the required performance of the suspension system. To design the controller in the inner loop, the model without the noise caused by the road profile and velocity of the car is used. The outer control loop then designed with an adaptive mechanism calculates the active control force to compensate for the vibrations caused by the road profile and velocity of the car. The control force is determined by the error between states of the reference model and states of suspension systems, the reference model is the model of closed loop with inner control loop without the noise. The simulation results implemented by using the practice date of the road profile show that the capability of oscillation decrease for ASS is quite efficient.
\end{abstract}

This is an open access article under the CC BY-SA license.

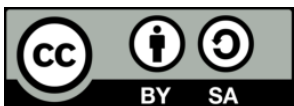

\section{Corresponding Author:}

Chi Nguyen Van,

Department of Control Engineering and Instruments,

Thai Nguyen University of Technology,

3/2 Street, Thai Nguyen City, Viet Nam.

Email:ngchi@tnut.edu.vn

\section{INTRODUCTION}

The main advantage of the active suspension system (ASS) is the capability of changing the dynamic wheel load (Fdyn) in order to improve the ride comfort for passengers by adjusting the characteristic of a suspension system like the stiffness, the damping. The electromagnetic, hydraulic actuators mostly are used to generate the active force in the ASS. The control problem for ASS is always more challenging for researching and application in the practice because of some reasons [1,2]: there are many ASS's states need to be controlled while ASS has only one control input, the controlled active force acts contemporaneously on both sprung mass and sprung mass, the performance bandwidth of actuator is limited, the limitations of time, the response time does not meet the requirement of high velocity, all ASS's states are not measured by sensors, the determining statistic characteristics of road excitation is the practice difficulty.

There are lots of control methods for ASS including: in the material [3], the authors used the selflearning neural networks to adjust controller parameters in order to improve the ride comfort, the complexity of neural networks and capabilities of convergence are the disadvantages of this method. Using the Skyhook reference model and the adaptive parameter controller based on the Lyapunov method is presented in [4]. In $[5,6]$, authors applied the free-model sliding mode control with time-delay estimation to eliminate the nonlinear, uncertainty and noise acting on the system. The modal feedback method used to control ASS is written in the material [7], this method control independently in each vibration mode of ASS, the controller parameters are turned by an optimal control method. Using second order optimal LQG controller for ASS is 
conducted in the [8], in which the ASS model is rewritten in the linear form. In the article [9], the controller based on backstepping method is used to control ASS, in this method control law is designed to back step from inner loop to outer loop by using the virtual control inputs. The simple decoupling method for ASS is implemented by the authors of [10]. Moreover, there are lots of control methods applied to ASS including the Event-triggered control [11], Ho control method using the Linear matrix inequality, the fuzzy logic control method [12], etc.

The common feature of the above methods is the noise caused by road profile considered to be the main noise input of the mathematical model, this model is not concern about that noise which is depend on the road profile and velocity of the car. In this paper, we use the general model of ASS, in that model the parameters related to the noise caused by road profile and velocity of the car are considered. From the practice test, we see that the faster velocity of the car is the small amplitude of the noise is and the faster frequency is. This feature makes the noise characteristic acting to the ASS change. Based on that model of ASS, the control process for ASS is implemented by two control loops. The inner control loop is designed with noises without affecting ASS, this control loop drives the performance of ASS in the transient period. The model of closed loop system with inner control loop is used as reference model for the designing the outer adaptive control loop. The reference model depends on the velocity of the car. The outer adaptive control loop uses the parameter adaptive mechanism adjusted by the velocity of the car and errors between ASS's states and states of the reference model. The adaptive force is generated to compensate for the above errors in order to drive that error reaching to zero. The simulation results using the practice road profile show that this method is efficient to apply to the practice. The remaining part of this paper is structured as follows: Part two mentions the modeling of the suspension system. Part three introduces adaptive control for the ASS. Part four shows the simulation results. Finally, part five summarizes some conclusions.

\section{MAIN CONTENTS}

\subsection{Modelling of the ASS}

The active suspension depicted in Figure 1 system consists of sprung mass $m_{s}$ and unsprung mass $m_{w}$, the sprung mass, and unsprung mass are connected by the suspension part including damping part $c_{s}$ and spring part $d_{s}$ in parallel [13]. In case of active suspension, the active actuator (electrical, electromagnetic, hydric devices driven by electrical signal $u(t)$ ) is added to this part to change the natural characteristics of suspension part in order to get the desired comfort ride. The connection between the unsprung mass and the road surface is the wheel. The wheel is described by wheel damping part $c_{w}$ and wheel spring part $d_{w}$. The states of the suspension system are deflection, velocity, and accelerator of sprung mass $z_{s}, \dot{z}_{s}, \ddot{z}_{s}$, unsprung mass $z_{w}, \dot{z}_{w} \ddot{z}_{w}$, respectively. The $z_{r}$ is the road excitation supported as the main input noise of the system, $z_{r}$ depends on the road profile and velocity of the car $v(t)$.

The performance quality of the suspension system is reflected upon the comfort ride, this quality is presented by 3 parameters: the accelerations of the car and the wheel $\ddot{z}_{s}, \ddot{z}_{w}$ respectively, the dynamic wheel load $F_{d}$ and the suspension deflection $z_{s}-z_{w}$. The dynamic wheel load is calculated as (1) as [14].

$$
F_{d}=c_{w}\left(z_{r}-z_{w}\right)+d_{w}\left(\dot{z}_{r}-\dot{z}_{w}\right)
$$

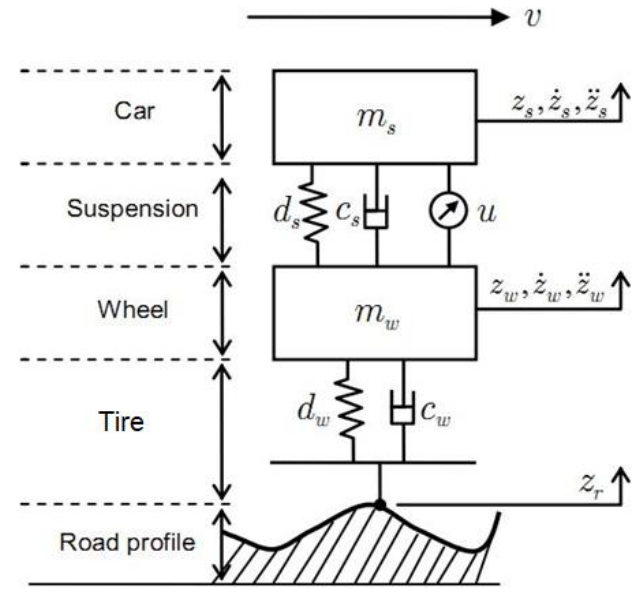

Figure 1. Physical model of a typical suspension system 
The dynamic wheel load $F_{d}$ depends on the mass of the car $m_{w}$ velocity of the car $v(t)$ and road profile. The smaller values of $\ddot{z}_{s}, \ddot{z}_{w}, F_{d}$ are, the higher comfort rider of the passengers in the car is Figure 1 . The road excitation always is supposed as the main input noise, but we realize that the $z_{r}$ not only depends on the road profile but also the velocity of the car. For the same road profile, as the velocity of the car changes, the stochastic characteristics of the $z_{r}$ will be changed.

Figure 2 shows the varying of the $z_{r}$ in the case velocity of the car changing from $5 \mathrm{~km} / \mathrm{h}$ to $45 \mathrm{~km} / \mathrm{h}$ for the same road profile. The road profile has the stochastic characteristics: $\min =-2.319 \mathrm{~cm}$, $\max =2.348 \mathrm{~cm}$, mean $=0.0131$, median $=0.0131 ;$ variance $=0.331678, \mu=0.0131, \sigma=0.576$. From Figure 3 we see that the velocity of the car is higher, the amplitude of the variation $z_{r}$ is lower. The maximum of $z_{r}$ is $0.07 \mathrm{ym}$ in the case $v(t)=5 \mathrm{~km} / \mathrm{h}$, the maximums of $z_{r}$ are $0.023 \mathrm{~m}$ and $0.02 \mathrm{~m}$ in the cases $v(t)=15 \mathrm{~km} / \mathrm{h}$ and $45 \mathrm{~km} / \mathrm{h}$, respectively. The changing of stochastic characteristics $z_{r}$ by the velocity of the car, the road profile will affect strongly to the state estimation process.

The first order model of the road excitation $z_{r}$ as the function of the road profile and velocity of the car depicted in Figure 4 can be written by the equation as $[15,16]$ :

$$
\frac{d z_{r}}{d t}=-\lambda v(t) z_{r}+w_{z r}
$$

where $\gamma, v$ are the optional feedback parameter and velocity of the car, respectively, $w_{z r}$ is the input noise caused by the road profile.

Let consider the state vector of the suspension system described in Figure 1 as:

$$
\begin{aligned}
& z_{1}=z_{s}, z_{2}=\dot{z}_{s}, \quad z_{3}=z_{w}, z_{4}=\dot{z}_{w}, \quad z_{5}=z_{r}, \quad z_{6}=\dot{z}_{r} \\
& \boldsymbol{z}=\left[\begin{array}{llllll}
z_{1} & z_{2} & z_{3} & z_{4} & z_{5} & z_{6}
\end{array}\right]^{T}
\end{aligned}
$$

Suppose $u(t)$ as the active force, if $u(t)=0$ system is called the passive suspension system. The model of the suspension system is written by the following equation.

$$
\frac{d \mathbf{z}}{d t}=\mathbf{A}(v(t)) \mathbf{z}+\mathbf{B} u(t)+\mathbf{w}(\mathrm{t})
$$

where

$$
\mathbf{A} \triangleq\left[\begin{array}{cccccc}
0 & 1 & 0 & 0 & 0 & 0 \\
\frac{-c_{s}}{m_{s}} & \frac{-d_{s}}{m_{s}} & \frac{c_{s}}{m_{s}} & \frac{d_{s}}{m_{s}} & 0 & 0 \\
0 & 0 & 0 & 1 & 0 & 0 \\
\frac{c_{s}}{m_{w}} & \frac{d_{s}}{m_{w}} & \frac{-c_{w-} c_{s}}{m_{w}} & \frac{-d_{w}-d_{s}}{m_{w}} & \frac{c_{w}-d_{w} \lambda v(t)}{m_{w}} & 0 \\
0 & 0 & 0 & 0 & 0 & 1 \\
0 & 0 & 0 & 0 & -\lambda v(t) & 0
\end{array}\right], \mathbf{B} \triangleq\left[\begin{array}{c}
0 \\
\frac{1}{m_{s}} \\
0 \\
1 \\
-\frac{m_{w}}{0} \\
0
\end{array}\right], \mathbf{w}(t)=\left[\begin{array}{c}
w_{z s} \\
w_{d z s} \\
w_{z w} \\
w_{d z w} \\
w_{z r} \\
w_{d z r}
\end{array}\right]
$$

By modifying the (5) as

$$
\frac{d \mathbf{z}}{d t}=\mathbf{A}(v(t)) \mathbf{z}+\widetilde{\boldsymbol{B}} u[\boldsymbol{\vartheta}(t)+\boldsymbol{d}(t)]
$$

with

$$
\widetilde{\mathbf{B}}=\left[\begin{array}{ll}
\mathbf{B} & I_{n}
\end{array}\right], \boldsymbol{\vartheta}(t)=\left(\begin{array}{c}
u(t) \\
\mathbf{0}_{n}
\end{array}\right), \boldsymbol{d}(t)=\left[\begin{array}{c}
0 \\
\mathbf{w}(t)
\end{array}\right], n=6
$$

the system has the input $u(t)$

$$
\boldsymbol{\vartheta}(t)=\left(\begin{array}{c}
u(t) \\
\mathbf{0}_{n}
\end{array}\right) \Rightarrow u(t)=\left[\begin{array}{ll}
1 & \mathbf{0}_{n}
\end{array}\right] \boldsymbol{\vartheta}(t)
$$

Matrix $\mathbf{A}(v(t))$ has six eigenvalues located in the left of the imaginary axis which make 03 pairs of eigenvalues that are symmetric with respect to the imaginary axis. Two first of them are fixed and have

The algorithm of adaptive control for active suspension systems using pole assign... (Chi Nguyen Van) 
the negative real part, the last pair depends on the velocity of the car. The velocity of the car increasing makes the imaginary part of the third pair of eigenvalues move along the imaginary axis far from the root as described in the Figure 3.

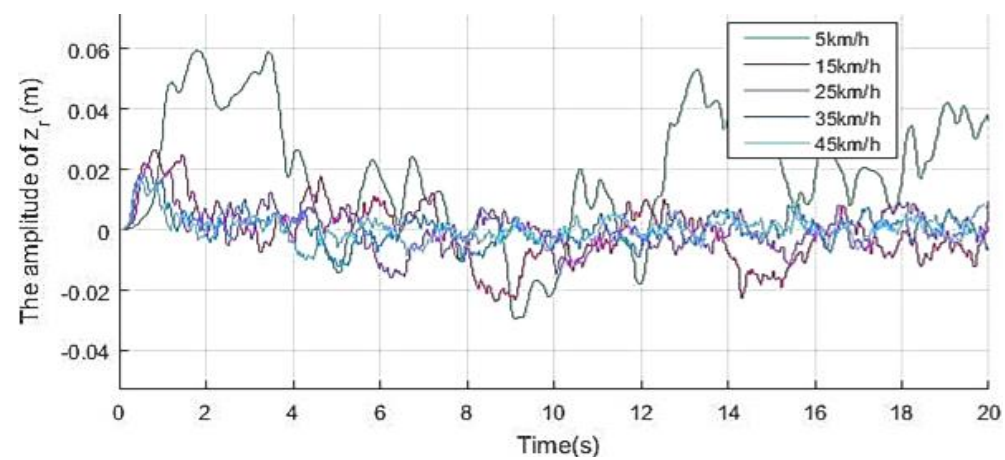

Figure 2. The varying of the stochastic characteristics for the same road profile by the velocity of the car

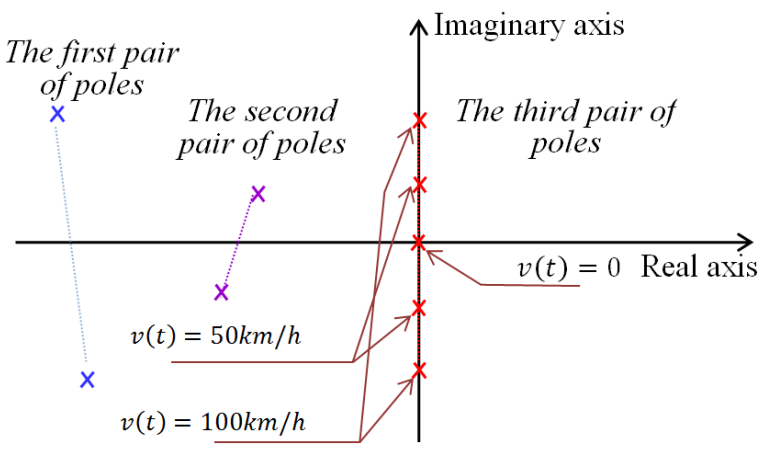

Figure 3. The locations of pole pairs of the ASS

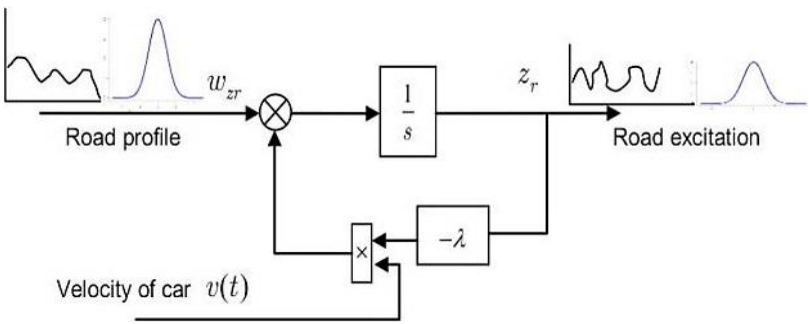

Figure 4. The first order model of road excitation $\mathrm{z}_{\mathrm{r}}$ as the function of the road profile and velocity of the car

\subsection{Adaptive control for the ASS}

The adaptive control for ASS is designed by two steps as follows.

- Step 1: Design the state feedback controller in order to keep closed loop system without noise $\boldsymbol{d}(t)=0$ having the desired dynamic. The pole placement is the technique that is used to place the closed loop poles of the system in predetermined locations. Based on the desired dynamic wheel load and the desired dynamic of $z_{w}, \dot{z}_{w}$ the predetermined locations of the poles are calculated. The results of Step 1 is the state feedback controller which has the gain depending on the velocity of the car and the road profile. This is the inner control loop.

- Step 2: Design the adaptive controller in the outer control loop to attenuate the noise caused by the road profile and velocity of the car. This control loop uses the reference model to be the model of inner loop without noise $\boldsymbol{d}(t)$, the dynamic of the reference model is depended on the velocity of the car and road 
profile. Using state error between the ASS and reference model, the adaptive mechanism calculates the active force to compensate for the noise caused by the road profile and velocity of the car. The adaptive mechanism gain is varied road condition and velocity of the car.

\subsubsection{Designing the inner control loop}

To design the controller in the inner control loop we suppose that the velocity of the car is zero, so $\mathbf{w}(t)=0$ or $\boldsymbol{d}(t)=\mathbf{0}_{n+1}$. Consider that all states of ASS $\boldsymbol{z}(\boldsymbol{t})$ are measurable. Therefore, the model of ASS can be written as (7):

$$
\frac{d \mathbf{z}}{d t}=\mathbf{A}(v(t)) \mathbf{z}+\widetilde{\mathbf{B}} \boldsymbol{\vartheta}(t)
$$

So the designing is to determine the matrix $\mathbf{R}(v(t))$ to guarantee that $\boldsymbol{\vartheta}(t)=\eta(t)+\mathbf{R}(v(t)) \mathbf{z}$ makes the model of closed-loop system become

$$
\frac{d \mathbf{z}}{d t}=(\mathbf{A}(v(t))-\widetilde{\mathbf{B} R}(v(t))) \mathbf{z}+\widetilde{\mathbf{B}} \eta(t)=\widetilde{\mathbf{A}}(v(t)) \mathbf{z}+\widetilde{\mathbf{B}} \eta(t)
$$

and the poles of the dynamic system $(8) s_{1}, s_{2}, \ldots, s_{n}$ are predetermined locations with respect to the desired performance of the ASS $[17,18]$. So, the $(9)$ needs to be solved.

$$
\operatorname{det}(s I-\mathbf{A}(v(t))-\widetilde{\mathbf{B} R}(v(t)))=\left(s-s_{1}\right)\left(s-s_{2}\right) \ldots\left(s-s_{1}\right)
$$

Note that the matrix $\mathbf{R}(v(t))$ is depended on the velocity of the car $v(t)$. One of the methods to solve (9) is the modal method in the material [19]. This method uses the regression calculation process. If $m$ is rank of matrix $\widetilde{\mathbf{B}}$ and $\lambda_{1}, \lambda_{2}, \ldots, \lambda_{n}$ are eigenvalues of matrix $\mathbf{A}$ then the regression calculation process is implemented by $\mathrm{n} / \mathrm{m}$ steps, on each step the matrix $\mathbf{R}_{k}$ is calculated to move $\mathrm{m}$ poles of A among $\lambda_{1}, \lambda_{2}, \ldots, \lambda_{n}$ to $m$ new poles among $s_{1}, s_{2}, \ldots, s_{n}$ of matrix $\mathbf{A}(v(t))-\widetilde{\mathbf{B}} \mathbf{R}(v(t))$. The matrix $\mathbf{R}(v(t))$ then is calculated by summing matrices $\mathbf{R}_{k}(v(t))$.

\subsubsection{Designing the outer adaptive control loop}

Back to the ASS in presence of noise $\mathbf{w}(t)$ caused by road profile and velocity of the car (6), with the inner control loop the ASS model becomes

$$
\frac{d \boldsymbol{z}}{d t}=\widetilde{\mathbf{A}}(v(t))+\widetilde{\mathbf{B}}[\eta(t)+\boldsymbol{d}(t)]
$$

in which $\widetilde{\mathbf{A}}$ is a stable matrix having desired poles $s_{1}, s_{2}, \ldots, s_{n}$. We have the Lyapunov (11)

$$
\widetilde{\mathbf{A}}(v(t))^{T} \mathbf{P}+\mathbf{P} \widetilde{\mathbf{A}}(v(t))=-\mathbf{Q}
$$

If symmetric matrix $\mathbf{Q}$ is positive definite, (11) always exists a unique positive definite symmetric matrix $\mathbf{P}(v(t))[20]$.

We use the reference model described by (12):

$$
\frac{d \mathbf{z}_{m}}{d t}=\widetilde{\mathbf{A}}(v(t)) \mathbf{z}_{m}+\widetilde{\mathbf{B}} \zeta(t)
$$

Designing the outer adaptive control loop is to guarantee the system (10) tracking to the system (12). Suppose $\boldsymbol{d}(t)=\boldsymbol{\theta}(t)$, we have

$$
\frac{d \mathbf{z}_{\boldsymbol{m}}}{d t}=\widetilde{\mathbf{A}}(v(t)) \mathbf{z}_{\boldsymbol{m}}+\widetilde{\mathbf{B}}(\zeta(t)+\boldsymbol{\theta}(t))
$$

So the task of noise attenuation adaptive mechanism is to adjust $\boldsymbol{\theta}(t)$ in order to make the error $\boldsymbol{e}(t)=\boldsymbol{z}(t)-\boldsymbol{z}_{\boldsymbol{m}}(t)$ tend to zero, in which 


$$
\frac{d \boldsymbol{e}}{d t}=\widetilde{\mathbf{A}}(v(t)) \boldsymbol{e}_{\boldsymbol{m}}+\widetilde{\mathbf{B}} \boldsymbol{\theta}(t)
$$

The diagram of two control loops for ASS is shown in the Figure 5.

Use the positive-definite Lyapunov candidate function as follows:

$$
V(e, \theta)=e^{T} P e+\theta^{T} H^{-1} \theta
$$

in which matrix $\boldsymbol{P}$ is root of Lyapunov function (15) and $\boldsymbol{H}$ is the positive-definite symmetric matrix chosen arbitrarily. So to keep $\frac{d V(\boldsymbol{e}, \boldsymbol{\theta})}{d t}<0$ or $\boldsymbol{e} \rightarrow \mathbf{0}$ for all $\boldsymbol{e} \neq \mathbf{0}$, the vector $\boldsymbol{\theta}$ needs to be satisfy (16)

$$
\left[\widetilde{\mathbf{B P}} \boldsymbol{e}+\boldsymbol{H}^{-\mathbf{1}} \frac{d \boldsymbol{\theta}}{d t}\right]=\mathbf{0}
$$

Therefore, the noise attenuation adaptive mechanism is

$$
\frac{d \boldsymbol{\theta}}{d t}=-\boldsymbol{H} \widetilde{\mathbf{B}}^{T} \mathbf{P}(v(t)) \boldsymbol{e}
$$

Some remarks:

- Function $\frac{d V(\boldsymbol{e}, \boldsymbol{\theta})}{d t}<0$ is negative-definite with respect to $\boldsymbol{e}$ therefore, $\boldsymbol{\theta} \neq \boldsymbol{d}$. In another word, the adaptive mechanism (19) is the noise attenuation mechanism, it is not an identification function of noise caused by road profile and velocity of the car.

- The velocity of tending to zero of error $\boldsymbol{e}$ is depended on the positive-definite matrix $\mathbf{Q}$.

- Larger the norm of matrix $\mathbf{H}$ is the faster error $\boldsymbol{e}$ tend to zero does. Larger eigenvalues of matrix $\mathbf{H}$ are the larger amplitude of $\boldsymbol{\theta}$ is, this causes the oscillation in the system

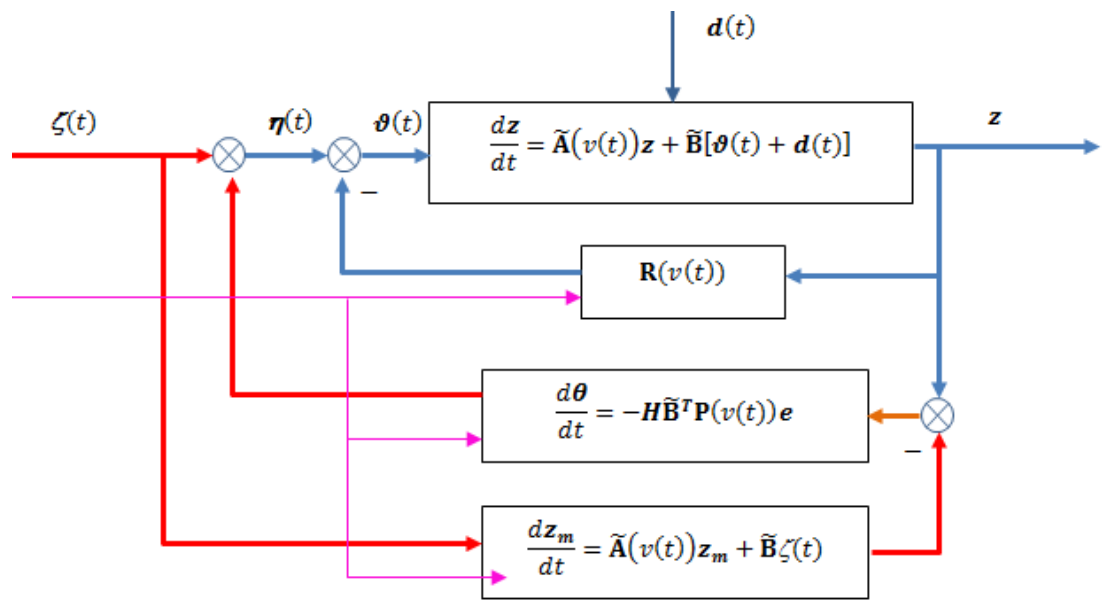

Figure 5. The diagram of two control loops for ASS: inner control loop (blue line), outer adaptive control loop (red line)

\subsubsection{The simulation results using the practical road profile data}

The simulation results below are conducted in two cases that are even road profile and stochastic practice road profile. The physical parameters of ASS are:

$$
\begin{aligned}
& \lambda=0.2, c_{s}=8399\left(\frac{N}{m}\right), c_{w}=151176\left(\frac{N}{m}\right), d_{c}=6665\left(\frac{N s e c}{m}\right), d_{w}=500\left(\frac{N s e c}{m}\right), \\
& m_{s}=194.2(\mathrm{~kg}), m_{w}=49(\mathrm{~kg})
\end{aligned}
$$

a. The simulation results for even road profile

The initial condition of states is [0;0.2;0;0.2], the amplitude of the event road profile is $0.02 \mathrm{~m}$ as described in Figure 6. The varying of position, the velocity of the sprung mass and unsprung mass are 
depicted in the Figure 7. In this figure, the passive case, the pole assign case, and the adaptive case are compared with. In the case of pole assign, the number of oscillations of position and velocity of sprung mass and unsprung mass is decreased but the amplitude of oscillation is increased in comparison to the passive case. In the case of adaptive active control, the amplitude and number of oscillations are both decreased.

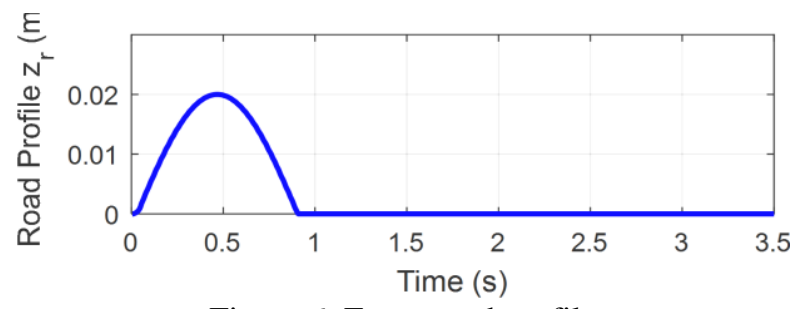

Figure 6. Event road profile
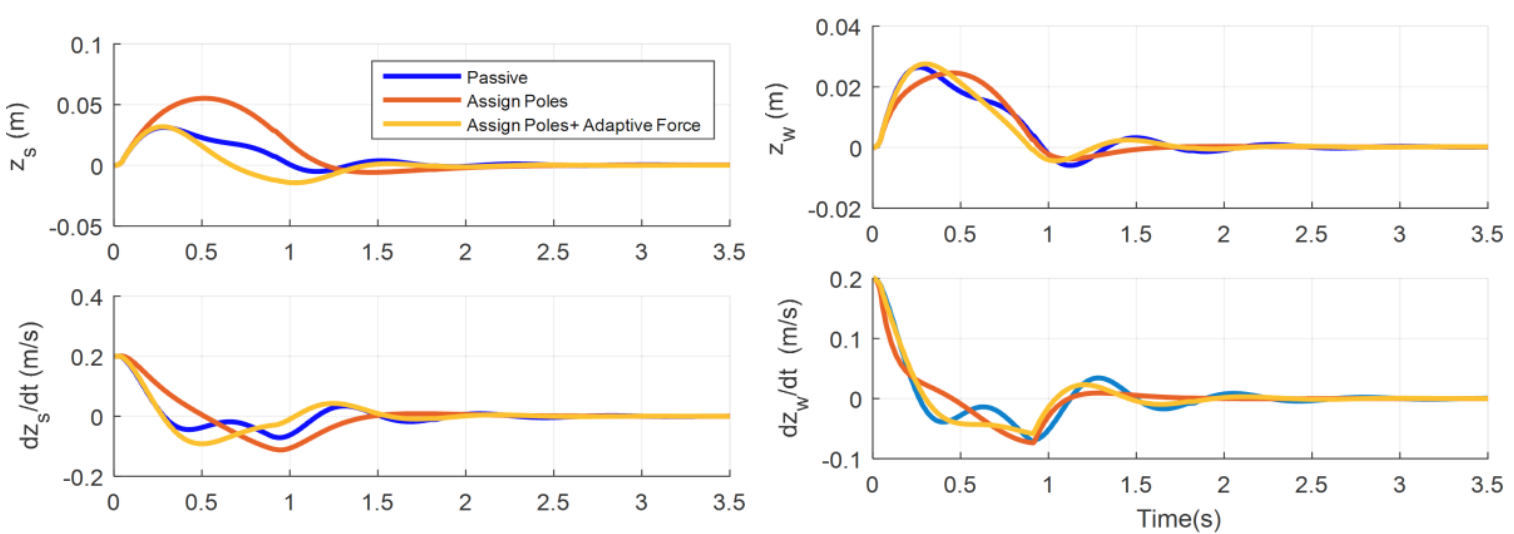

Figure 7. Response of position, velocity of the sprung mass and unsprung mass in the case of event road profile

b. The simulation results for stochastic practice road profile

Probability density function of the stochastic practice road profile (as in Figure 8) is: $\min =-2.319 \mathrm{~cm}, \max =2.348 \mathrm{~cm}$, mean $=0.0131$, median $=0.0131 ;$ variance $=0.331678, \mu=$ 0.0131 . The simulation results are conducted for three velocities of the car $5 \mathrm{~km} / \mathrm{h}, 40 \mathrm{~km} / \mathrm{h}$ and $80 \mathrm{~km} / \mathrm{h}$. Figures 9 and 10 are depicted the simulation oscillation responses of ASS, the dynamic wheel load and active force, respectively. In these figures, the active control responses are compared to the passive case. Similarly, Figures 11 and 12 show the simulation results in the case that the velocity of the car is $40 \mathrm{~km} / \mathrm{h}$, Figures 13 and 14 plot the results for $80 \mathrm{~km} / \mathrm{h}$.

At $5 \mathrm{~km} / \mathrm{h}$ of the velocity of the car, we see that the oscillation and amplitude of oscillation the sprung mass is effectively decreased as shown in the Figure 9. The active control force acts to the system clearly at $0.5 \mathrm{~s}$ from the beginning. Efficiency of the oscillation decrease of the car is better than the wheel. Reducing car oscillation is more efficient than reducing the wheel oscillation. The dynamic load varies in range $\pm 300 \mathrm{~N}$, the active control force ranges from $-600 \mathrm{~N}$ to $400 \mathrm{~N}$.

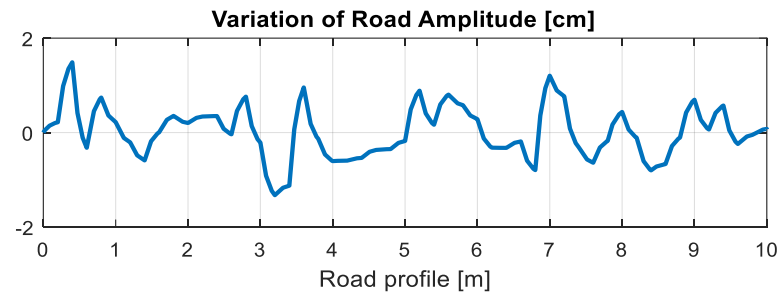

Figure 8. Stochastic practice road profile 

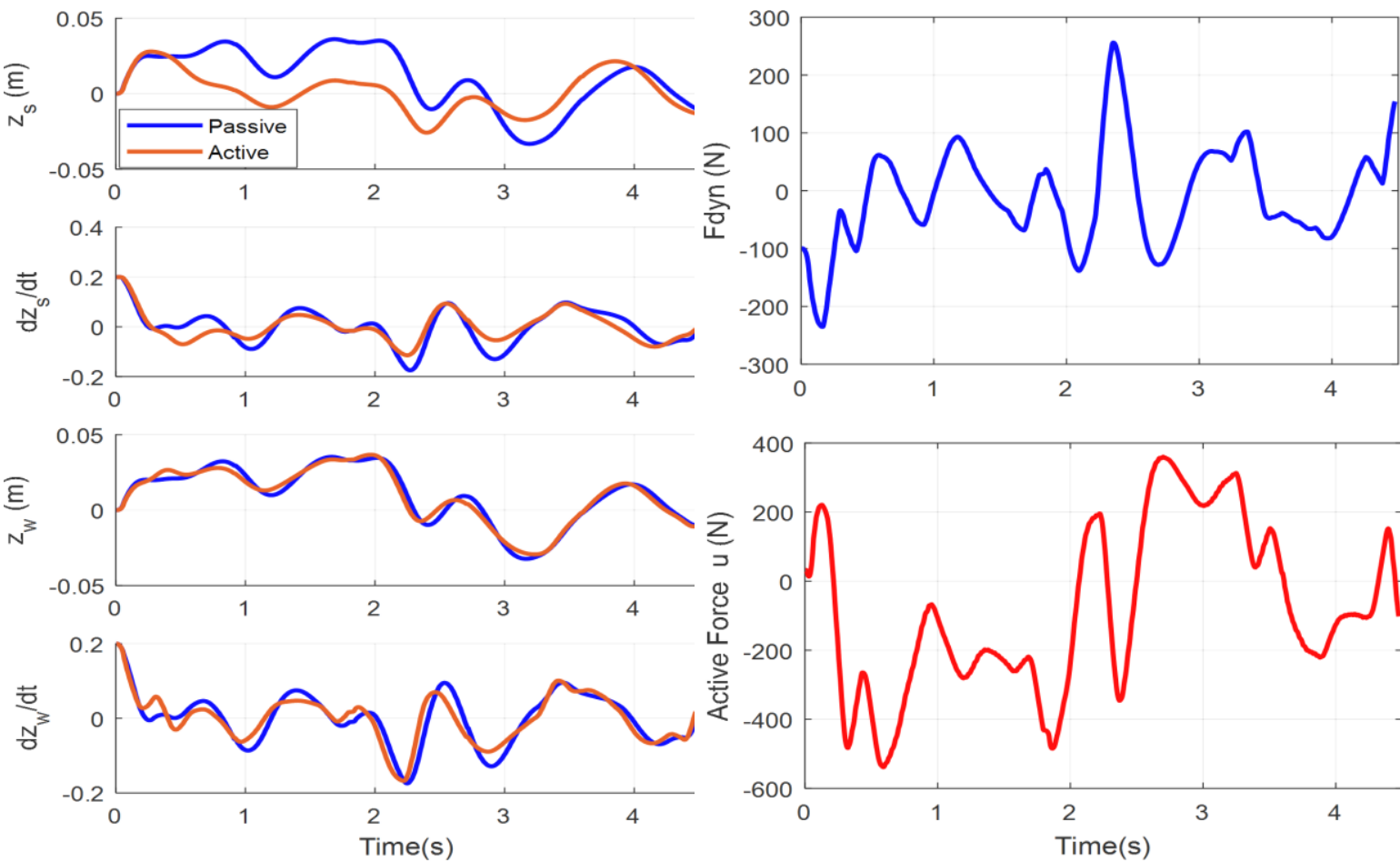

Figure 9. Position and velocity of sprung mass and unsprung mass for cases: passive and adaptive control at $5 \mathrm{~km} / \mathrm{h}$ (velocity of the car)

Figure 10. The dynamic wheel load and adaptive control force at $5 \mathrm{~km} / \mathrm{h}$ (velocity of the car)
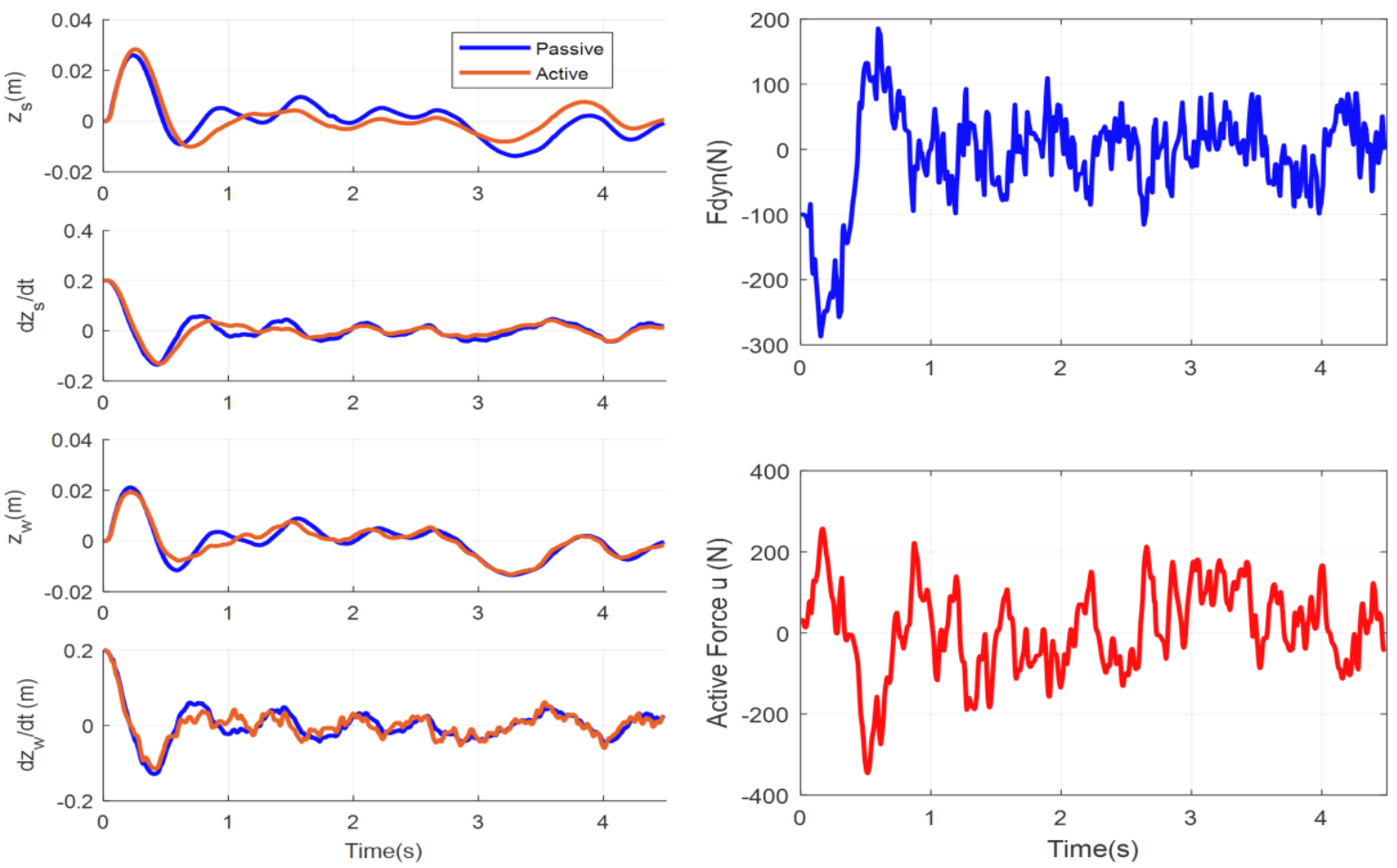

Figure 11. Position and velocity of sprung mass and unsprung mass for cases: passive and adaptive control at $40 \mathrm{~km} / \mathrm{h}$ (velocity of the car)

Figure 12. The dynamic wheel load and adaptive control force at $40 \mathrm{~km} / \mathrm{h}$ (velocity of the car) 

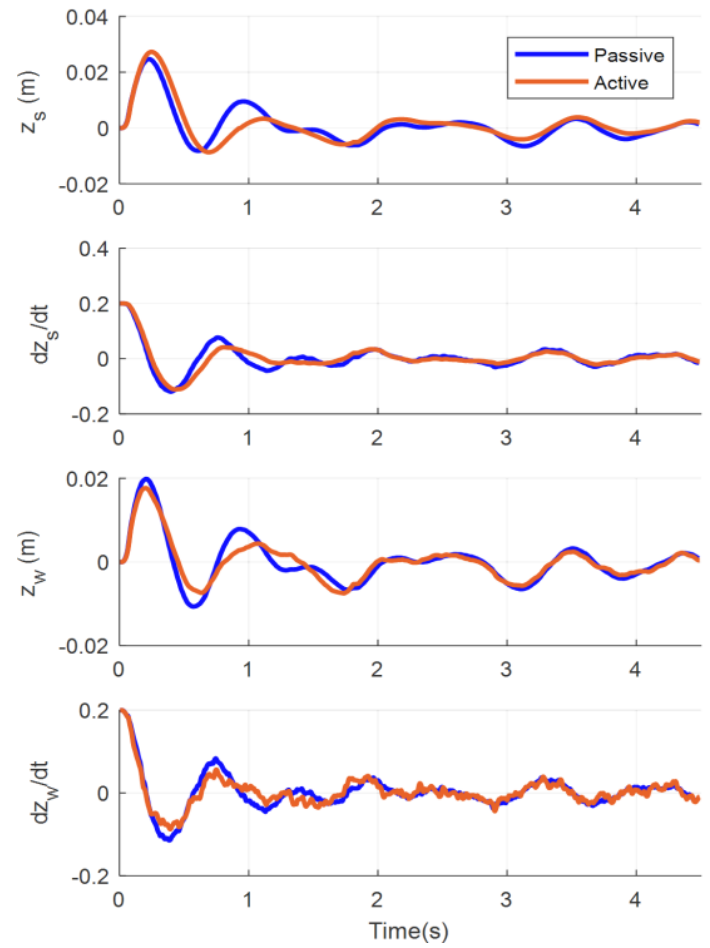

Figure 13. Position and velocity of sprung mass and unsprung mass for cases: passive and adaptive control at $80 \mathrm{~km} / \mathrm{h}$ (velocity of the car)
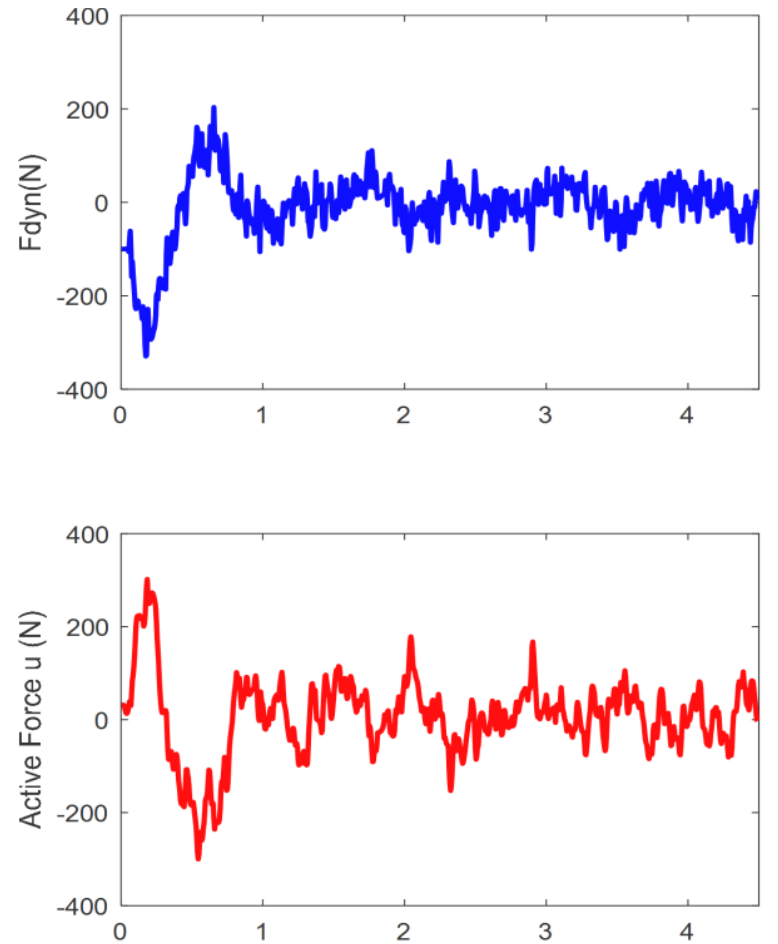

Figure 14. The dynamic wheel load and adaptive control force at $80 \mathrm{~km} / \mathrm{h}$ (velocity of the car)

At car speed of $40 \mathrm{~km} / \mathrm{h}$ with active adaptive control, the car oscillation decrease is quite good in comparison to the passive case. For the first 0.5 seconds of the adaptive control process, the dynamic wheel load varies in the range $-300 \mathrm{~N}$ to $200 \mathrm{~N}$, and after that the dynamic wheel load decreases, it ranges in $\pm 100 \mathrm{~N}$. The active control force varies from $400 \mathrm{~N}$ to $400 \mathrm{~N}$ for the first 0.5 seconds and it varies in the range $\pm 200 \mathrm{~N}$ for after that. At car speed of $80 \mathrm{~km} / \mathrm{h}$, the simulation results are the same at $40 \mathrm{~km} / \mathrm{h}$. Therefore, by using the cascade adaptive control for the ASS, the oscillations of the car and the wheel are decreased effectively, it is the same case with the dynamic load.

\section{CONCLUSION}

The paper introduces the cascade adaptive control method for an ASS. The inner control loop uses the pole placement technique which is used to place the closed loop poles of the system without noise in predetermined locations in order to change the dynamic characteristics of ASS to desired performance requirements. The outer control loop uses the adaptive control strategy with the adaptive mechanism to compensate for the oscillation of the car caused by the road profile and velocity of the car acting to ASS. The simulation results using even road profile and stochastic practice road profile show that the capability of oscillation decrease for the car and the wheel of ASS is quite efficient. The dynamic wheel load is decreased so the ride comfort of passengers is better.

\section{ACKNOWLEDGEMENTS}

This research is supported financially by Thai Nguyen University of Technology, TNUT.

\section{REFERENCES}

[1] D. Karnopp, "Theoretical Limitations in Active Vehicle Suspensions," Vehicle System Dynamics, vol. 15, no. 1, pp. 41-54, 1986.

[2] M. C. Smith, "Achievable Dynamic Response for Automotive Active Suspensions," Vehicle System Dynamics, vol. 24, no. 1, pp. 1-33, 1995

[3] J. Lin and R. Lian, "Intelligent Control of Active Suspension Systems," IEEE Transactions on Industrial Electronics, vol. 58, no. 2, pp. 618-628, 2011. 
[4] A. Turnip, et al., "Controller design for active suspension system based on skyhook reference model," in 2015 International Conference on Technology, Informatics, Management, Engineering \& Environment (TIME-E), 2015, pp. 147-151.

[5] V. S. Deshpande, M. Bhaskara, and S. B. Phadke, "Sliding mode control of active suspension systems using a disturbance observer," in 2012 12th International Workshop on Variable Structure Systems, 2012, pp. 70-75.

[6] W. Jue and Z. Jing, "Model-free tracking control for vehicle active suspension systems," in 2015 34th Chinese Control Conference (CCC), 2015, pp. 8067-8072.

[7] F. Braghin, F. Resta, and E. Sabbioni, "A modal control for active/semi-active suspension systems," in 2007 IEEE/ASME international conference on advanced intelligent mechatronics, 2007, pp. 1-6.

[8] B. Zhang, M. Fan, and F. Miao, "Optimal Control of Vehicle Active Suspension Systems with Actuator Delay," in 2007 IEEE International Conference on Control and Automation, 2007, pp. 2257-2261.

[9] A. Alleyne and J. K. Hedrick, "Nonlinear Control of a Quarter Car Active Suspension," in 1992 American Control Conference, 1992, pp. 21-25.

[10] G. Tang, H. Lin, and H. Su, "Decoupling vibration control for active suspension systems," in 2017 29th Chinese Control and Decision Conference (CCDC), 2017, pp. 6504-6509.

[11] K. Bansal and P. Mukhija, "Event-triggered control of vehicle active suspension systems," in 2018 Indian Control Conference (ICC), 2018, pp. 178-183.

[12] S.-j. Liu, Z.-h. Huang, and Y.-z. Chen, "Automobile active suspension system with fuzzy control," Journal of Central South University of Technology, vol. 11, no. 2, pp. 206-209, 2004.

[13] C. N. Van, "State Estimation Based on Sigma Point Kalman Filter for Suspension System in Presence of Road Excitation Influenced by Velocity of the Car," Journal of Control Science and Engineering, vol. 2019, 2019.

[14] G. Koch and T. Kloiber, "Driving State Adaptive Control of an Active Vehicle Suspension System," in IEEE Transactions on Control Systems Technology, vol. 22, no. 1, pp. 44-57, 2014.

[15] D. Hrovat, "Survey of advanced suspension developments and related optimal control applications," Automatica, vol. 33, no. 10, pp. 1781-1817, 1997.

[16] G. Koch, et al., "A nonlinear estimator concept for active vehicle suspension control," in Proceedings of the 2010 American Control Conference, 2010, pp. 4576-4581.

[17] K. Ogata, Modern Control Engineering. Prentice Hall PTR, 2001.

[18] C. N. Van and P. N. Doan, "Adaptive tracking control based on disturbance attenuation and ISS stabilization of Euler-Lagrange nonlinear systems in the presence of uncertainty and input noise," in 2011 2nd International Conference on Artificial Intelligence, Management Science and Electronic Commerce (AIMSEC), 2011, pp. 36983701.

[19] G. Yao, et al., "Modal computing method of output feedback control gain matrix in pole assignment of vibration system," in 2010 International Conference on Mechanic Automation and Control Engineering, 2010, pp. 22432247.

[20] G. Kitagawa, "An algorithm for solving the matrix equation X = FXF T + S," International Journal of Control, vol. 25, no. 5, pp. 745-753, 1977.

Int J Rob \& Autom, Vol. 9, No. 4, December 2020 : $271-280$ 